\title{
A predictive screening tool to detect diabetic retinopathy or macular edema in primary health care: construction, validation and implementation on a mobile application
}

Cesar Azrak, Antonio Palazón-Bru, Manuel V Baeza-Díaz, David Folgado De la Rosa, Carmen Hernández-Martínez, José j MartínezToldos, Vicente F Gil-Guillén

The most described techniques used to detect diabetic retinopathy and diabetic macular edema have to be interpreted correctly, such that a person not specialized in ophthalmology, as is usually the case of a primary care physician, may experience difficulties with their interpretation; therefore we constructed, validated and implemented as a mobile app a new tool to detect diabetic retinopathy or diabetic macular edema (DRDME) using simple objective variables. We undertook a cross-sectional, observational study of a sample of 142 eyes from Spanish diabetic patients suspected of having DRDME in 2012-2013. Our outcome was DRDME and the secondary variables were: type of diabetes, gender, age, $\mathrm{HbAlc}$, foveal thickness and visual acuity (best corrected). The sample was divided into two parts: $80 \%$ to construct the tool and $20 \%$ to validate it. A binary logistic regression model was used to predict DRDME. The resulting model was transformed into a scoring system. The area under the ROC curve (AUC) was calculated and risk groups established. The tool was validated by calculating the AUC and comparing expected events with observed events. The construction sample $(n=106)$ had 35 DRDME (95\% Cl: 24.1-42.0\%), and the validation sample $(n=36)$ had 12 DRDME (95\% Cl: 17.9 48.7\%). Factors associated with DRDME were: $\mathrm{HbAlc}$ (per 1\%) $(\mathrm{OR}=1.36,95 \% \mathrm{Cl}: 0.93-$ 1.98, $p=0.113)$, foveal thickness (per $1 \mu \mathrm{m})(\mathrm{OR}=1.03,95 \% \mathrm{Cl}: 1.01-1.04, p<0.001)$ and visual acuity (per unit) $(\mathrm{OR}=0.14,95 \% \mathrm{Cl}: 0.00-0.16, p<0.001)$. AUC for the validation: 0.90 (95\% Cl: $0.75-1.00, p<0.001$ ). No significant differences were found between the expected and the observed outcomes $(p=0.422)$. In conclusion, we constructed and validated a simple rapid tool to determine whether a diabetic patient suspected of having DRDME really has it. This tool has been implemented on a mobile app. Further validation studies are required in the general diabetic population. 
1 Authors: Cesar Azrak [1,2], Antonio Palazón-Bru [2,3], Manuel Vicente Baeza-Díaz [1], David

2 Manuel Folgado-de la Rosa [2], Carmen Hernández-Martínez [1], José Juan Martínez-Toldos

3 [1], Vicente Francisco Gil-Guillén [2,3].

4 Institutions:

5 1. Ophthalmology Service, General Hospital of Elche, Elche, Alicante, Spain.

6 2. Clinical Medicine Department, Miguel Hernández University, San Juan de Alicante, Alicante, 7 Spain.

8 3. Research Unit, Elda Hospital, Elda, Alicante, Spain.

9 Corresponding author: Prof. Antonio Palazón-Bru, PhD, Department of Clinical Medicine,

10 Miguel Hernández University, San Juan de Alicante, Spain. E-mail: antonio.pb23@gmail.com.

11 Phone: +34 965919449. Fax: +34 965919450. 


\section{3}

14

15

16

17

\section{INTRODUCTION}

Diabetes mellitus is prevalent worldwide and its most important ophthalmological complications are diabetic retinopathy and diabetic macular edema (Jeganathan, Wang \& Wong, 2008; International Diabetes Federation, 2013). The main consequences of these disorders are possible loss of vision and blindness (Jeganathan, Wang \& Wong, 2008). Considering that these conditions can be treated in their initial stages it is important to diagnose them as early as possible and thus prevent the dire consequences (No authors listed, 1991; No authors listed, 1995).

The Valencian Community, an autonomous Mediterranean region in southeast Spain, has a population of some 5 million (Instituto Nacional de Estadística, 2014). A diabetic patient in this Community is mainly controlled by the primary care team, who refer the patient to the ophthalmological specialist when the patient reports sudden loss of visual acuity, is suspected of having macular edema, proliferative retinopathy, advanced retinopathy is shown on nonmydriatic retinography screening or there is evidence of cataracts (Generalitat Valenciana: Conselleria de Sanitat, 2006). This protocol is based on relevant scientific literature to treat diabetic retinopathy and maculopathy, for both primary health care and ophthalmology services. Thus, the diabetic patient has the diabetic retinopathy monitored independently of its severity. It is regularly managed by the primary care physician during its early stages and by ophthalmologists in more severe cases (Generalitat Valenciana: Conselleria de Sanitat, 2006).

The most described techniques used to detect diabetic retinopathy are non-mydriatic retinography and biomicroscopy of the retina (No authors listed, 1991; Baeza et al., 2009; Ryan et al., 2013), and those used to detect diabetic macular edema include biomicroscopy, angiography and optical coherence tomography (No authors listed, 1995; Ryan et al., 2013). 
However, most of these tests have to be interpreted correctly, such that a person not specialized in ophthalmology, as is usually the case of a primary care physician, may experience difficulties with their interpretation, as this is subjective. Accordingly, we undertook a study in the Valencian Community aimed at constructing and validating by means of a mathematical model a tool to detect diabetic retinopathy and diabetic macular edema using simple objective variables, which would therefore present no interpretation difficulties. In addition, as the mathematical model requires arithmetic operations, the tool has been implemented on a mobile app. The results of this study, therefore, provide a simple tool to help primary care services determine whether a diabetic patient needs to be referred to the ophthalmological specialist.

\section{METHODS}

\section{Study population}

The population study involved diabetic patients followed by the ophthalmological service of the General University Hospital of Elche (Valencian Community, Spain). These patients are referred from the primary health care services, according to the protocol (Generalitat Valenciana: Conselleria de Sanitat, 2006).

\section{Study design and participants}

This cross-sectional, observational study was undertaken in a sample of diabetic patients referred to the ophthalmological service of the General University Hospital of Elche by the primary care teams between October 2012 and June 2013 and who were willing to participate. The sampling procedure consisted of randomly selecting one day each week (not always the same day) and recruiting all the diabetic patients who attended that day, by means of linear systematic sampling. A patient was considered to have diabetes if the diagnosis had been made by a 
physician (ICD9-MC 250.X). Patients were excluded if they had dementia, high myopia or another macular disorder, had had vitreoretinal surgery, a cataract operation during the previous 3 months, had received laser treatment in the macular area or panphotocoagulation, or were taking anti-angiogenic drugs.

\section{Variables and measurements}

The main outcome variable was the presence of at least treatable diabetic retinopathy (severe, very severe or proliferating (Ryan et al., 2013)) or diabetic macular edema (DRDME). The diagnosis of these two disorders was made by clinical ophthalmological examination of the retina by indirect ophthalmoscopy and biomicroscopy of the central retina with a Topcon slit lamp, model SL-8Z (Topcon Corporation; Tokyo, Japan) using a 78 diopter lens (78D aspheric lens. Volk Optical Incorporated Company; Mentor, $\mathrm{OH}$ ) and indirect ophthalmoscopy with a 28D lens by an expert retinal ophthalmologist. Macular edema was defined as the presence of hard exudates or localized retinal thickening within a distance of $500 \mu \mathrm{m}$ from the fovea, and the degree of diabetic retinopathy was defined according to the ETDRS study (No authors listed, 1991). The secondary variables were type of diabetes, gender, age (years), glycated hemoglobin (HbAlc) (\%), foveal thickness $(\mu \mathrm{m})$ and visual acuity (best corrected). The source of information for data concerning the type of diabetes, hypertension, dyslipidemia, gender, smoking, age and $\mathrm{HbAlc}$ was the clinical history. Best corrected visual acuity was obtained using the Snellen scale. The foveal thickness was obtained at the central area by dilating the pupil with a drop of tropicamide and measuring with spectral domain optical coherence tomography (Topcon 3D OCT 2000; Topcon Corporation ${ }^{\circledR}$, Itabashi, Tokyo, Japan). The images were acquired by 512 horizontal linear scans and 128 vertical scans, centered on the fixation point making a $6 \times 6$ square 3D pattern. The mean retinal thickness was calculated automatically by the software of the 
82 device. We measured a $6 \mathrm{~mm}$ diameter area, centered on the fovea, thereby using for the study

83 the central $1000 \mu \mathrm{m}$ area (the central circle).

84 Sample size

85 The construction sample involved data from 106 patients, of which 35 had DRDME. To contrast

86 an area under the ROC curve (AUC) different to 0.5 , assuming a 95\% confidence level and

87 expecting to find an AUC of 0.9 , the contrast power was nearly $100 \%$. Using the same

88 parameters in the validation sample (36 patients, 12 with DRDME), we obtained a contrast

89 power of $97.17 \%$ (Hanley \& McNeil, 1982).

90 Statistical methods

91 The quantitative variables are reported as means and standard deviations, and the qualitative

92 variables as absolute and relative frequencies. All the analyses were done with a significance of

$935 \%$ and the confidence interval was calculated for each relevant parameter. The complete sample

94 was divided into two parts. One part $(80 \%)$ to construct the predictive model and the other part

$95(20 \%)$ to validate the model constructed.

96 Construction: we used a binary logistic regression model to predict DRDME using the

97 most clinically relevant variables, taking into account that we could only use a maximum number

98 of explanatory variables in the model (10 observations of our outcome for each explanatory

99 variable). The resulting model was transformed into a scoring system using the method of the

100 Framingham study (Sullivan, Massaro \& D'Agostino, 2004). The ROC curve was calculated and

101 we constructed groups based on the probabilities of the model: low $(<25 \%)$, medium $(25-50 \%)$,

102 high (50-75\%) and very high ( $\geq 75 \%)$.

103 In the validation sample the AUC was calculated and the observed events compared with

104 the expected events of the model using the $\mathrm{X}^{2}$ test. No calculations were made of the classical 
105 indicators of a diagnostic test, such as sensitivity, specificity, predictive values and likelihood

106 ratios, as the test constructed does not indicate a single value (positive or negative) but rather a

107 probability of DRDME associated with each score. Accordingly, differences were studied

108 between the expected (given by the predictive model) and the observed events to determine

109 whether the reality corresponded to what was indicated by the model. On the other hand,

110 calculating the AUC indicates the discriminating power of our model. A similar methodology

111 has been used in other studies (Palazón-Bru et al., 2015).

112 Mobile application

113 The models were implemented on a mobile application for the operating systems Android and

114 iPhone (see Supplemental Note S1). This application is free to download from any of the stores.

115 Its name is Diabetic retinopathy predictor.

116 Ethical consideration

117 The study was approved by the Ethics Committee of the General Hospital of Elche. All the

118 patients signed the informed consent document. The study was undertaken in accordance with

119 the basic principles of the World Medical Association Declaration of Helsinki and complied with

120 the norms described in the European Union guidelines for good clinical practice.

\section{RESULTS}

123 Table 1 shows the information for the two samples. In the Construction sample $(n=106)$ there

124 were 35 cases of DRDME (95\% CI: 24.1-42.0\%) (retinopathy alone, 8; macular edema alone, 9;

125 both disorders, 18). In the Validation sample $(n=36)$ there were 12 cases of DRDME $(95 \% \mathrm{CI}$ :

126 17.9-48.7\%), of which 6 had both disorders, 3 just macular edema and 3 just retinopathy. For the

127 other characteristics, in both samples there was a majority of type 2 diabetes (76.5-85.7\%), an 
128 older mean age (62.8-63.4 years), high HbA1c (7.7-7.9\%), visual acuity of 0.7 and mean foveal

129 thickness between 261.2 and $285.2 \mu \mathrm{m}$. No significant differences were found between the two

130 samples (p: 0.117-0.886).

131 The factors associated with DRDME (Table 1) were: HbA1c (per 1\%) $(\mathrm{OR}=1.36,95 \%$

132 CI: 0.93-1.98, p=0.113), foveal thickness (per $1 \mu \mathrm{m})(\mathrm{OR}=1.03,95 \% \mathrm{CI}: 1.01-1.04, \mathrm{p}<0.001)$

133 and visual acuity (per unit) $(\mathrm{OR}=0.14,95 \% \mathrm{CI}: 0.00-0.16, \mathrm{p}<0.001)$. The scoring system with its

134 risk groups created from these factors is shown in Fig. 1. In the model we selected the three most

135 clinically relevant variables, as we had 35 outcomes in our sample (Alasil et al., 2010; Buabbud,

136 Al-latayfeh \& Sun, 2010; Hermann et al., 2014; Varma et al., 2014; Palazón-Bru et al., 2015).

137 In the Validation sample (Fig. 2) the AUC was 0.90 (95\% CI: $0.75-1.00, \mathrm{p}<0.001)$. No

138 significant differences were found between the expected outcomes and the observed outcomes $139(\mathrm{p}=0.422)($ Fig. 3).

140

141 DISCUSSION

142 This study constructed an innovative tool able to determine whether a diabetic patient has

143 diabetic retinopathy or diabetic macular edema, and who should therefore be referred to a

144 specialist in ophthalmology. The model uses easily obtained variables and the calculation can be

145 done easily and simply using a mobile application.

146 A literature search revealed no such multifactor models determining the likelihood of

147 presenting DRDME, so that it is not possible to compare our model overall. However, we can

148 make independent comparisons with the factors obtained. We found greater complications with

149 higher levels of Hb1 Ac and a greater foveal thickness, and a lower likelihood of DRDME as the 
150 visual acuity decreased. These particular findings are consistent with others (Alasil et al., 2010;

151 Buabbud, Al-latayfeh \& Sun, 2010; Hermann et al., 2014; Varma et al., 2014).

This study constructed and validated a tool to enable the physician to discriminate

153

154

155

156

157

158

159

160

161

162

163

164

165

166

167

168

169

170

171

172

between those patients who should be referred to an ophthalmologist and those who do not need to be. Thus, the primary care physician should carry out regular control examinations to assess the possible need for referral. A patient found to be in the High or Very High group should be referred to the specialist, as the associated likelihood of DRDME is very high. On the other hand, if a patient is found to be in the Medium or Low groups ( $<3$ points), the primary care physician should control the patient. A model such as the one explained herein generally presents certain difficulties in its use, as the model is usually complex and requires a certain time to do the calculation. However, our model uses very simple variables that, once introduced into the mobile application, give the likelihood of the outcome. Finally, a nursing professional who receives adequate training (use of the apparatus and measurement of visual acuity) could also use the proposed model and thus reduce the workload of the primary care physician.

In some regions optical coherence tomography is not used in primary health care. Nevertheless, consideration could be given to its use in order to improve the screening of referable retinopathy. This tool, though, is expensive, so it is not feasible to include it at all health centers. An instrument could, though, be purchased for each health care area (each area covers various health care centers), and diabetic patients suspected as having referable retinopathy could attend this particular center to undergo the test described here. This method would reduce the possible cost considerably. Another possibility would be the use of a mobile unit that could rotate between the various health centers. This would help to integrate our predictive model into usual clinical practice, with the idea being to refer to the specialist services 
173 just those patients who really need it. Additionally, the inclusion of optical coherence

174 tomography in primary health care could enable other macular disorders to be monitored, as this

175 device can provide greater information about the macula. The resulting information, though,

176 should be interpreted by an ophthalmologist, whereas the foveal thickness is a completely

177 objective measure that can be interpreted with the other clinical parameters of our model by a

178 healthcare professional working in the area of primary care. Finally, this study could be

179 undertaken by other healthcare systems with different referral criteria, with the idea of reducing

180 the proportion of patients who really need to be referred to the specialist ophthalmological

181 services yet still following their own protocols.

182 Strengths and limitations of the study

183 The main strength of this study concerns its novel way to construct and validate a scoring system

184 to help primary care physicians take decisions about referring a diabetic patient to the

185 ophthalmological specialist. Additionally, the factors used in the model are obtained objectively, 186 so they do not have to be interpreted, as is the case with retinography.

187 Although the sample size may seem small, it is sufficient for the aims of the study

188 (constructing and validating the model), as the contrast power was greater than 95\%, whereas

189 this is generally $80-90 \%$. There are, too, non-significant variables in the model constructed.

190 However, as in other studies, we have to consider that we are evaluating the model overall and

191 not variable by variable, that is the goodness of fit (likelihood ratio test $=53.4, \mathrm{p}<0.001$;

192 Nagelkerke $\mathrm{R}^{2}=0.583$ ) and the AUC in the validation sample (0.90) (Palazón-Bru et al., 2015).

193 As this model was validated in diabetic patients who had already been referred to the specialist,

194 further studies are needed in the general diabetic population to determine if it is applicable as a

195 screening test for all diabetic patients when they attend their primary health care center (selection 
196 bias). These would study the probabilities of DRDME with our scoring system, as the general

197 population would have a low prevalence of this macular disorder. On the other hand, concerning

198 measurement bias, all the measurements were taken using calibrated devices and in accordance

199 with current guidelines. Finally, we did not include other variables in the model that could have

200 influenced DRDME, such as the duration of the disease. This particular variable was not

201 assessed because the clinical history does not record it and the patient knows the duration of the

202 disease only approximately. This could therefore cause an information bias, which is why it was

203 not included in this study. Nevertheless, even without this variable the predictive model had an

204 AUC of 0.90 , which equates to a great discriminating power.

205

206

CONCLUSION

207 This study constructed and validated a predictive model based on a scoring system to determine

208 whether a diabetic patient referred to the specialist has DRDME. This model can be used to

209 reduce the volume of patients referred to the ophthalmological services from primary health care

210 centers. Nevertheless, further studies should be undertaken to determine whether the model is

211 applicable as a screening test in the general diabetic population.

212

\section{ACKNOWLEDGMENTS}

214 We thank Ian Johnstone for help with the English language version of the manuscript. 


\section{REFERENCES}

216 Alasil T, Keane PA, Updike JF, Dustin L, Ouyang Y, Walsh AC, Sadda SR. 2010. Relationship

217 between optical coherence tomography retinal parameters and visual acuity in diabetic macular

218 edema. Ophthalmology 117:2379-2386.

Baeza M, Orozco-Beltrán D, Gil-Guillen VF, Pedrera V, Ribera MC, Pertusa S,

Merino J. 2009. Screening for sight threatening diabetic retinopathy using non-mydriatic retinal camera in a primary care setting: to dilate or not to dilate? International Journal of Clinical Practice 63:433-438.

Buabbud JC, Al-latayfeh MM, Sun JK. 2010. Optical coherence tomography imaging for diabetic retinopathy and macular edema. Current Diabetes Reports 10:264-269.

Generalitat Valenciana: Conselleria de Sanitat. 2006. Plan Diabetes Comunidad Valenciana

2006-2010 Available at http://publicaciones.san.gva.es/publicaciones/documentos/V.4928-

$230 \quad$ 2006.pdf (accessed October 2014).

231

232 Hermann JM, Hammes HP, Rami-Merhar B, Rosenbauer J, Schütt M, Siegel E, Holl

RW; DPV Initiative the German BMBF Competence Network Diabetes Mellitus. 2014. HbA1c

234 variability as an independent risk factor for diabetic retinopathy in type 1 diabetes: a

235 German/Austrian multicenter analysis on 35,891 patients. PLoS One 9:e91137.

236 
237 Hanley JA, McNeil BJ. 1982. The meaning and use of the area under a receiver operating

238 characteristic (ROC) curve. Radiology 143:29-36.

239

240 International Diabetes Federation.2013. IDF Diabetes Atlas. 6th ed. IDF.

Jeganathan VS, Wang JJ, Wong TY. 2008. Ocular associations of diabetes other than diabetic retinopathy. Diabetes Care 31:1905-1912. Review.

No authors listed. 1991. Early photocoagulation for diabetic retinopathy. ETDRS report number

9. Early Treatment Diabetic Retinopathy Study Research Group. Ophthalmology 98(5

Suppl):766-785.

No authors listed. 1995. Focal photocoagulation treatment of diabetic macular edema.

Relationship of treatment effect to fluorescein angiographic and other retinal characteristics at baseline: ETDRS report no. 19. Early Treatment Diabetic Retinopathy Study Research Group. Archives of Ophthalmology 113:1144-1155.

Palazón-Bru A, Martínez-Orozco MJ, Perseguer-Torregrosa Z, Sepehri A, Folgado-de la Rosa validation of a model to predict nonadherence to guidelines for prescribing antiplatelet therapy to hypertensive patients. Current Medical Research and Opinion 31:883-889. 
259 Ryan SJ, Schachat AP, Wilkinson CP, Hinton DR, Sadda SR, Wiedemann P. 2013. Retina. 5th 260 ed. China: EISEVIER.

261

262 Sullivan LM, Massaro JM, D'Agostino RB Sr. 2004. Presentation of multivariate data for clinical 263 use: The Framingham Study risk score functions. Statistics in Medicine 23:1631-1660. Review. 264

265 Varma R, Bressler NM, Doan QV, Gleeson M, Danese M, Bower JK, Selvin E, Dolan C, Fine J, 266 Colman S, Turpcu A. 2014. Prevalence of and risk factors for diabetic macular edema in the 267 United States. JAMA Ophthalmology 132:1334-1340.

268 
1

Scoring system to predict diabetic retinopathy and diabetic macular edema.

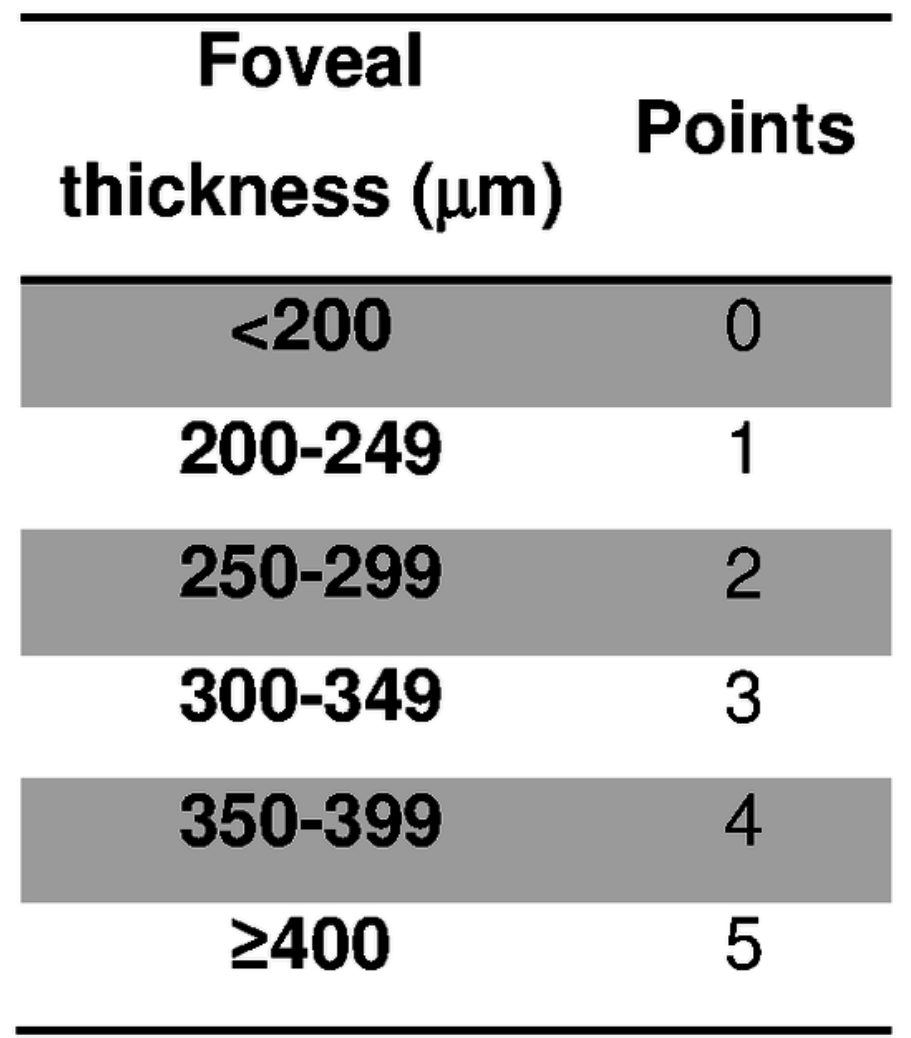

$\overline{\text { HbA1c (\%) Points }}$

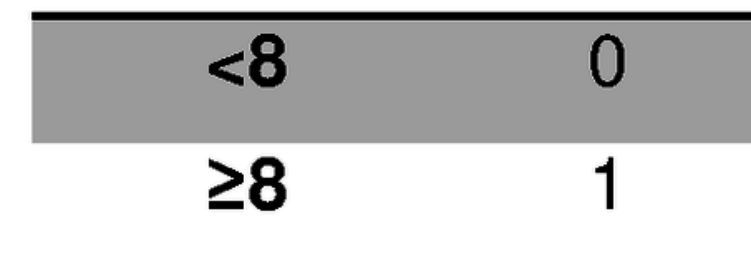

\section{Visual acuity Points}

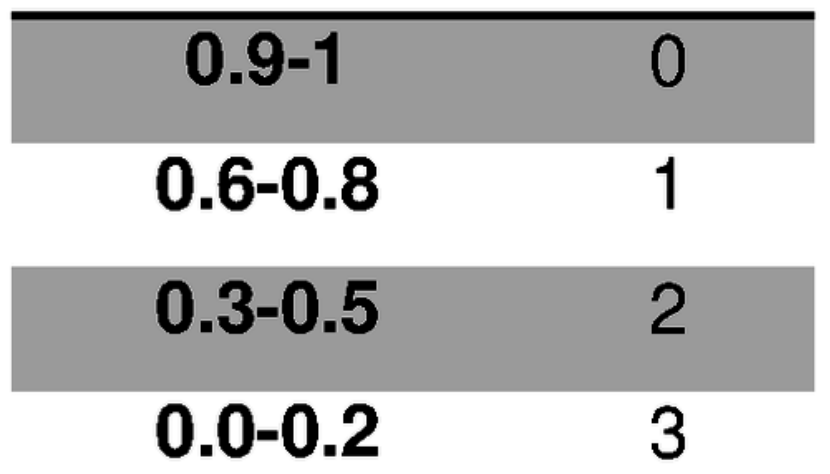

Points Sum Risk (\%)

\begin{tabular}{cc}
\hline Low (0-2) & $1.45-15.35$ \\
\hline Medium (3) & $39.15-39.20$ \\
High (4) & $69.55-69.60$ \\
Very high ( $\geq 5)$ & $\geq 89.05$ \\
\hline
\end{tabular}


2

Area under the ROC curve of the scoring system.

AUC, area under the ROC curve; $\mathrm{Cl}$, confidence interval.

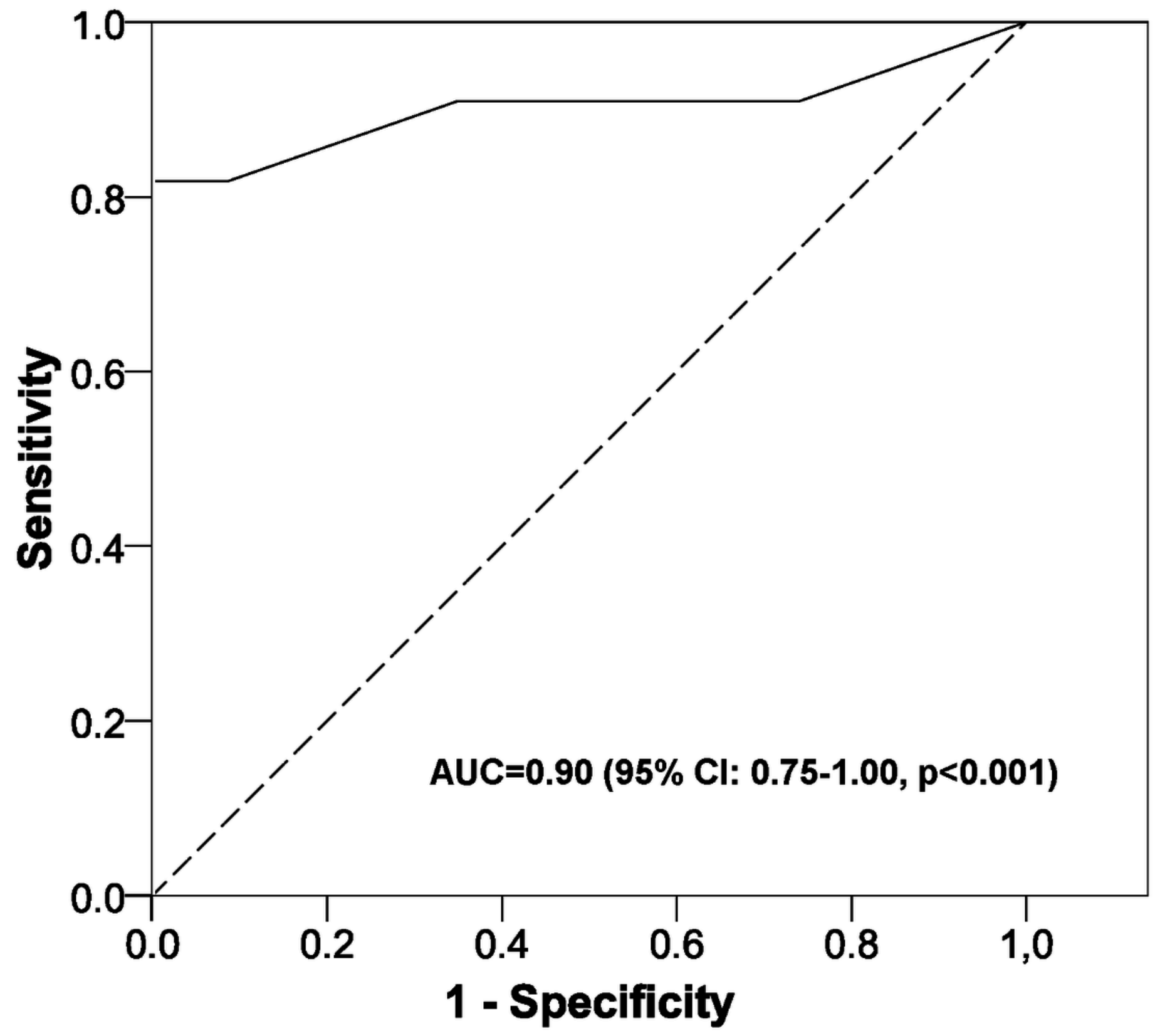


3

Validation of the scoring system.

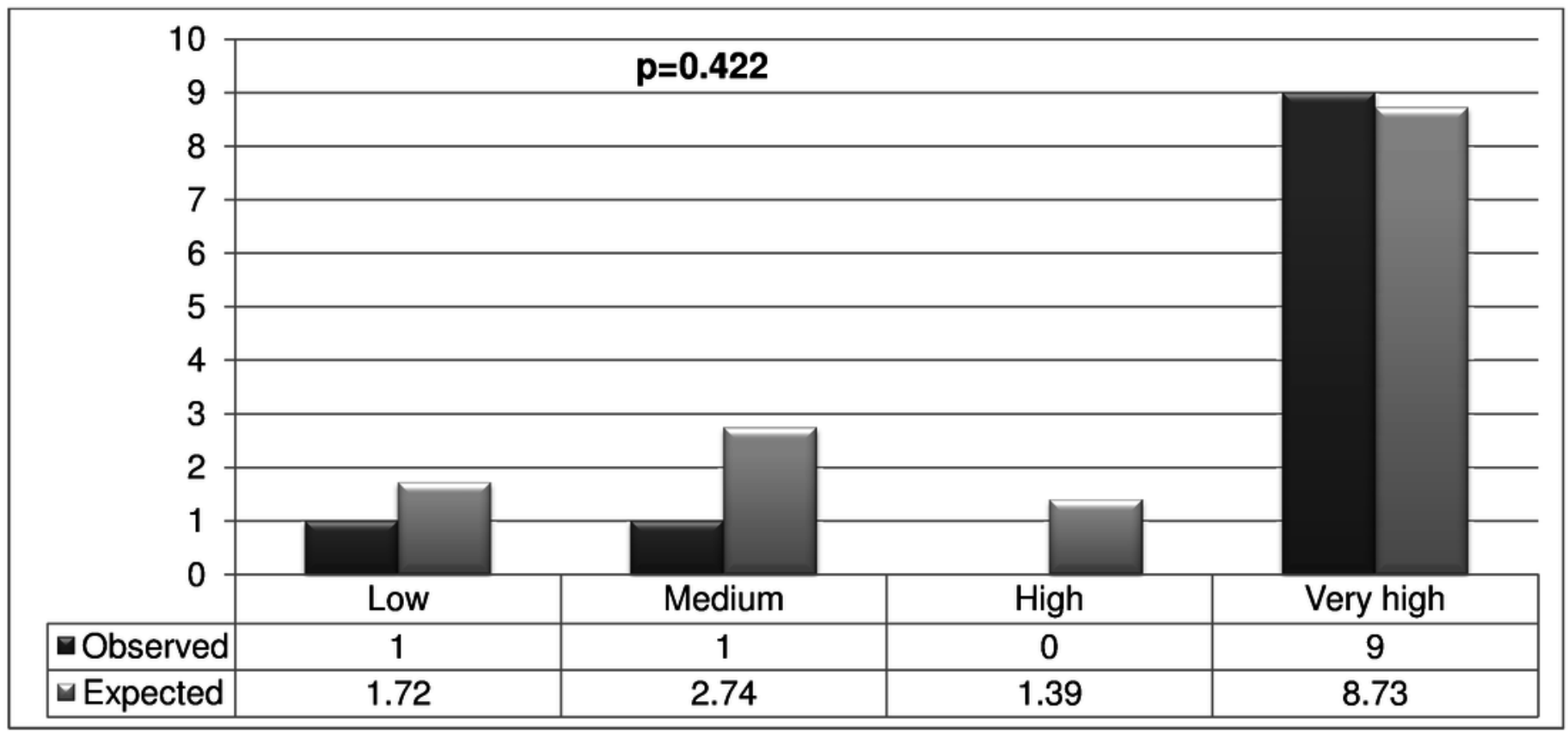




\section{Table $\mathbf{1}$ (on next page)}

Descriptive characteristics and analysis for diabetic retinopathy or macular edema in diabetic patients from a Spanish region. 2012-2013 data.

Abbreviations: Adj. OR, adjusted odds ratio; $\mathrm{Cl}$, confidence interval; DM, diabetes mellitus;

DRDME, diabetic retinopathy or diabetic macular edema; N/A, not applicable; N/M, not in the model. $n(\%)$, absolute frequency (relative frequency); $x \pm s$, mean \pm standard deviation. Goodness-of-fit: likelihood ratio test $=53.4, p<0.001$; Nagelkerke $R^{2}=0.583$. 


\begin{tabular}{|c|c|c|c|c|c|}
\hline Variable & 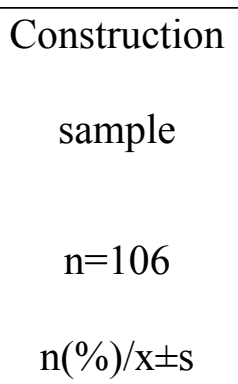 & $\begin{array}{l}\text { Validation } \\
\text { sample } \\
\mathrm{n}=36 \\
\mathrm{n}(\%) / \mathrm{x} \pm \mathrm{s}\end{array}$ & $\begin{array}{c}\mathrm{p}- \\
\text { value }\end{array}$ & $\begin{array}{l}\text { Adj. OR for } \\
\text { DRDME } \\
(95 \% \mathrm{CI})\end{array}$ & $\begin{array}{c}\mathrm{p}- \\
\text { value }\end{array}$ \\
\hline DRDME & $35(34.7)$ & $12(33.3)$ & 0.886 & $\mathrm{~N} / \mathrm{A}$ & $\mathrm{N} / \mathrm{A}$ \\
\hline DM Type 2 & $90(85.7)$ & $26(76.5)$ & 0.207 & $\mathrm{~N} / \mathrm{M}$ & $\mathrm{N} / \mathrm{M}$ \\
\hline Female gender & $52(49.1)$ & $20(55.6)$ & 0.500 & $\mathrm{~N} / \mathrm{M}$ & $\mathrm{N} / \mathrm{M}$ \\
\hline Age (years) & $63.4 \pm 14.4$ & $62.8 \pm 16.8$ & 0.847 & $\mathrm{~N} / \mathrm{M}$ & $\mathrm{N} / \mathrm{M}$ \\
\hline HbA1c (\%) & $7.7 \pm 1.5$ & $7.9 \pm 1.8$ & 0.643 & $\begin{array}{c}1.36 \\
(0.93-1.98)\end{array}$ & 0.113 \\
\hline $\begin{array}{l}\text { Foveal } \\
\text { thickness }(\mu \mathrm{m})\end{array}$ & $261.2 \pm 71.3$ & $285.2 \pm 95.1$ & 0.117 & $\begin{array}{c}1.03 \\
(1.01-1.04)\end{array}$ & $<0.001$ \\
\hline Visual acuity & $0.7 \pm 0.3$ & $0.7 \pm 0.3$ & 0.689 & $\begin{array}{c}0.14 \\
(0.00-0.16)\end{array}$ & $<0.001$ \\
\hline
\end{tabular}

1 